International Journal of Computational and Applied Mathematics.

ISSN 1819-4966 Volume 12, Number 2 (2017), pp. 511-523

(C) Research India Publications

https://dx.doi.org/10.37622/IJCAM/12.2.2017.511-523

\title{
Find Critical Eigenvalue in the Rayleigh-Bénard- Brinkman Convection Problem with Boundary Conditions by using Advanced SLM
}

\author{
Dr. Ashoka S.B. \\ B. MCA., Ph.D. \\ Department of Computer Science, MSCWB \\ Bangalore-560 080, India.
}

\begin{abstract}
An eigen boundary value problem is solved for the critical eigenvalue in the case of a Rayleigh-Bénard-Brinkman convection problem bounded by permeable walls with Robin boundary condition on temperature and general boundary condition on velocity. Unknown initial values and guess eigenvalue required to initiate the advanced successive linearization method are obtained using the single-term Rayleigh-Ritz method. The local thermal nonequilibrium between the solid and fluid phases in the Newtonian fluidsaturated porous medium results in coupled partial differential equations. It is shown that five steps of the method results in a convergent solution with an accuracy of 6 decimal digits.
\end{abstract}

Keywords: porous medium, local thermal non-equilibrium, convection, linear stability, advanced successive linearization method

\section{INTRODUCTION}

Convection in porous medium usually known as the Horton-Rogers-Lapwood problem has been the focus of interest of many researchers over the past several decades. The study initiated by Horton and Rogers [1] and Lapwood [2] is a porous medium version of Rayleigh-Bénard problem in a clear fluid layer and has wide variety of practical applications. These include oil reservoir modeling, geothermal engineering, building thermal insulation, grain storage and nuclear waste repository 
and so on. A comprehensive study on the convection in porous media is well documented in the books Ingham and Pop [3], Nield and Bejan [4] and Vafai [5]. Most of the studies in literature concerning Horton-Rogers-Lapwood problem assume that there is no temperature difference between the fluid and solid phases, at any location of the porous medium system. This is called as local thermal equilibrium (LTE) model. In many practical situations LTE model fails to comprehend the heat transport in porous medium. Hence it is important to assume that the solid and fluid phases are not in local thermal equilibrium by considering two-field model of the energy equation and this model is popularly non as the local thermal non-equilibrium (LTNE) model in the literature. Heat pipes, cooling of computer chips, food processing, microwave heating are few applications where LTNE effects are predominant. Vowing to these applications there have been number of works in literature concerning the LTNE model. Banu and Rees [6] were the first amongst others to study the onset of Darcy-Bénard convection using a LTNE model and obtained the critical Rayleigh number as a function of local thermal non-equilibrium parameters. Postelnicu and Rees [7] extended the work of Banu and Rees [6] to Darcy-Brinkman-Bénard convection by considering a form-drag term, using a thermal non-equilibrium with stress-free boundaries. It was shown that the inclusion of quadratic drag term in the momentum equation has no effect on stability criteria since the basic state being motionless. Further, they recovered LTE results by performing asymptotic analysis for large inter-phase heat transfer coefficient $H$. Malashetty, et. al. [8] studied the onset of Lapwood-Brinkman convection using a LTNE model and reiterated the results obtained by Postelnicu and Rees [7]. Nouri-Borujerdi et. al. [9] studied the effect local thermal non-equilibrium on conduction in a fluid saturated porous channel with a uniform heat source and obtained conditions under which the LTNE can be assumed. Postelnicu [10] extended the earlier work by Postelnicu and Rees [7] to rigid boundaries and gave a quantitative comparison between the results of stress-free and rigid boundaries. Above-cited works concern Newtonian liquid as a working medium. In recent past non-Newtonian fluids are being used in place of their Newtonian counterparts as most of the industrially important fluids exhibit nonNewtonian characteristics. With this view point researchers have extended the use of LTNE effects in non-Newtonian fluid saturated porous medium. For example, Shivakumara et. al. [11] studied the onset of convection in a viscoelastic fluidsaturated sparsely packed porous layer using a thermal non-equilibrium model. They showed that viscoelastic effects enhance stabilizing effect of the interphase heat transfer coefficient. Recently, Malashetty et. al. [12] studied the effect of thermal nonequilibrium on the onset of convection in a couple stress fluid saturated porous layer and showed that interphase heat transfer coefficient increases the critical Rayleigh number for the onset of convection with the increasing values of couple stress parameter. In literature there are works addressing the LTNE on the double diffusive convection in a fluid saturated porous layer. Double diffusive convection in a porous 
layer using a thermal non-equilibrium model was studied by Malashetty et. al. [13] and they showed that interphase heat transfer coefficient has a significant say on the stationary, oscillatory and finite amplitude double diffusive convection in a fluid saturated porous medium. Malashetty and Heera [14] and Malashetty et. al. [15] performed linear and non-linear stability analyses of the double diffusive convection in a rotating porous layer (tightly/sparsely packed) using a thermal non-equilibrium model. In these studies it was shown that the rotation increases the stabilizing effect of interphase heat transfer coefficient in all the three modes of double diffusive convection, namely, stationary, oscillatory and finite amplitude. Works of Malashetty et. al. [16] and Shivakumara et. al. [17] takes into account of thermal non-equilibrium effects on convective instability in an anisotropic porous layer. In all the studies mentioned so far concerning LTNE either stress-free or rigid boundaries with isothermal conditions are made use. But, the interface conditions (slip conditions) and the thermal boundary conditions of the third type are the most general and practical boundary conditions. To the best of authors knowledge there are no works concerning Rayleigh-Bénard-Brinkman convection with LTNE and two boundary condition. Hence, we propose to investigate the same in the present paper.

\section{MATHEMATICAL FORMULATION}

We consider a layer of fluid saturated porous medium of depth $d$ which is heated from below and cooled from above, as depicted in Figure 1. The upper surface is held at a temperature $T_{0}$ while the lower one is at $T_{0}+\Delta T$. It is assumed that both form-drag and boundary effects are significant, that the porous medium is isotropic but that local thermal equilibrium does not apply. Thus the governing equations, i.e., the continuity, momentum and energy equations, subject to the Boussinesq approximation, take the form

$$
\begin{gathered}
\nabla \cdot \vec{q}=0, \\
\frac{\rho_{f}}{\varepsilon}\left(\frac{\partial \vec{q}}{\partial t}+\frac{1}{\varepsilon} \vec{q} \cdot \nabla \vec{q}\right)=-\nabla p+\mu_{e} \nabla^{2} \vec{q}-\frac{\mu_{f}}{K} \vec{q} \\
+\rho_{f} \beta g\left(T_{f}-T_{0}\right) \hat{k}, \\
\varepsilon(\rho c)_{f} \frac{\partial T_{f}}{\partial t}+(\rho c)_{f} \vec{q} \cdot \nabla T_{f}=\varepsilon k_{f} \nabla^{2} T_{f}+h\left(T_{S}-T_{f}\right), \\
(1-\varepsilon)(\rho c)_{s} \frac{\partial T_{s}}{\partial t}=(1-\varepsilon) k_{s} \nabla^{2} T_{s}-h\left(T_{S}-T_{f}\right),
\end{gathered}
$$




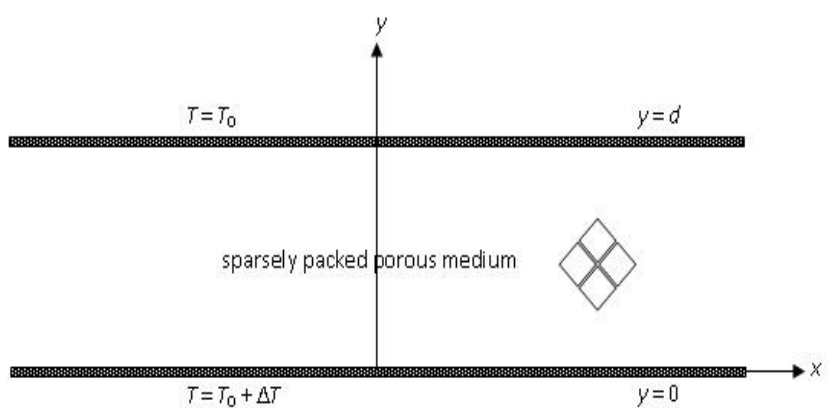

Fig.1. Schematic diagram of the physical configuration.

where, $\vec{q}=(u, v)$ is the velocity vector, $t$ is time, $\rho$ is the density, $\varepsilon$ is the porosity of the porous, medium, $p$ is the pressure, $\mu_{e}$ is the effective viscosity, $K$ is the permeability of the porous medium, $\beta$ is the coefficient of thermal expansion, $g$ is acceleration due to gravity, $c$ is specific heat at constant pressure, $T$ is the temperature, $k$ is the thermal conductivity, $h$ is the inter-phase heat transfer coefficient. Further, the properties of fluid phase are indicated with the subscript $f$ and those of solid phase are denoted by the subscript $s$.

The following velocity boundary conditions at the permeable walls are made use (see, Siddheshwar [18]):

$$
\left.\begin{array}{llll}
v=0, & \frac{\partial u}{\partial y}=\frac{\alpha_{1}}{\sqrt{K}} u & \text { at } & y=0 \\
v=0, & \frac{\partial u}{\partial y}=\frac{-\alpha_{2}}{\sqrt{K}} u & \text { at } & y=d .
\end{array}\right\}
$$

Here, $\alpha_{1}$ and $\alpha_{2}$ are dimensionless quantities depending on the material parameters which characterizes the structure of the permeable boundary membranes. We define the following dimensionless variables 


$$
\left.\begin{array}{l}
\left(x^{*}, y^{*}\right)=\frac{1}{d}(x, y), \quad t^{*}=\frac{(\rho c)_{f} d^{2}}{k_{f}} t, \\
\vec{q}^{*}=\left(u^{*}, v^{*}\right)=\frac{\varepsilon k_{f}}{(\rho c)_{f} d}(u, v)=\frac{\varepsilon k_{f}}{(\rho c)_{f} d} \vec{q}, \\
p^{*}=\frac{\mu_{e} k_{f}}{(\rho c)_{f} K} p, \Theta=\frac{T_{f}-T_{0}}{\Delta T}, \quad \Phi=\frac{T_{s}-T_{0}}{\Delta T} .
\end{array}\right\}
$$

Using (6) in equations (1) - (4) and dropping asterisks for simplicity, we obtain

$$
\nabla \cdot \vec{q}=0
$$

$$
\begin{gathered}
\frac{D a}{\varepsilon \operatorname{Pr}}\left(\frac{\partial \vec{q}}{\partial t}+\vec{q} \cdot \nabla \vec{q}\right)=-\frac{1}{\varepsilon} \nabla p+\nabla^{2} \vec{q}-\vec{q}+R a \Theta \hat{j}, \\
\frac{\partial \Theta}{\partial t}+\vec{q} \cdot \nabla \Theta=\nabla^{2} \Theta+H(\Phi-\Theta) \\
M \frac{\partial \Phi}{\partial t}=\nabla^{2} \Phi-\gamma H(\Phi-\Theta) .
\end{gathered}
$$

The non-dimensional numbers appearing in equations (7) - (10) are:

$$
\begin{aligned}
& D a=\frac{\mu_{e}}{\mu_{f}} \frac{K}{d^{2}} \text { the Darcy number, } \\
& \operatorname{Pr}=\frac{\mu_{e}(\rho c)_{f}}{\rho_{f} k_{f}} \text { the Prandtl number, } \\
& R a=\frac{\rho_{f} g \beta K d \Delta T}{\varepsilon \mu_{f} k_{f}} \text { the Darcy-Rayleigh number, } \\
& H=\frac{h d^{2}}{\varepsilon k_{f}} \text { the inter-phase heat transfer parameter, } \\
& \gamma=\frac{\varepsilon k_{f}}{(1-\varepsilon) k_{s}} \text { the porosity-scaled conductivity ratio and } \\
& M=\frac{(\rho c)_{s}}{(\rho c)_{f}} \frac{k_{f}}{k_{s}} \text { the diffusivity ratio. }
\end{aligned}
$$


The usual Rayleigh number, which is based on the mean properties of the porous medium, is given by

$$
R a^{*}=\frac{\gamma}{1+\gamma} R
$$

We now introduce the steam function $\Psi(x, y)$ that takes care of continuity equation (7) as follows

$$
u=-\frac{\partial \Psi}{\partial y}, \quad v=\frac{\partial \Psi}{\partial x} .
$$

We substitute equation (11) into equations (8) - (10) and eliminating pressure from the momentum equation we get the following equations:

$$
\begin{gathered}
\frac{D a}{\varepsilon \operatorname{Pr}}\left\{\frac{\partial}{\partial t}\left(\nabla^{2} \Psi\right)+\frac{\partial\left(\Psi, \nabla^{2} \Psi\right)}{\partial(x, y)}\right\}=D a \nabla^{4} \Psi \\
-\nabla^{2} \Psi+R a \frac{\partial \Theta}{\partial x}, \\
\frac{\partial \Theta}{\partial t}+\frac{\partial(\Psi, \Theta)}{\partial(x, y)}=\nabla^{2} \Theta+H(\Phi-\Theta), \\
M \frac{\partial \Phi}{\partial t}=\nabla^{2} \Phi-\gamma H(\Phi-\Theta) .
\end{gathered}
$$

The basic state is assumed to be quiescent and the heat transport takes place through conduction alone and hence we have

$$
\Psi_{b}=0, \quad \Theta_{b}=1-y, \quad \Phi_{b}=1-y .
$$

We now superimpose a small perturbation on the basic state as follows

$$
\Psi=\bar{\psi}, \quad \Theta=1-y+\bar{\theta}, \quad \Phi=1-y+\bar{\varphi} .
$$


Substituting equation (16) in equations (12) - (14) and linearizing we obtain

$$
\begin{gathered}
\frac{D a}{\varepsilon \operatorname{Pr}} \frac{\partial}{\partial t}\left(\nabla^{2} \bar{\psi}\right)=D a \nabla^{4} \bar{\psi}-\nabla^{2} \bar{\psi}+R a \frac{\partial \bar{\theta}}{\partial x}, \\
\frac{\partial \bar{\theta}}{\partial t}+\frac{\partial \bar{\psi}}{\partial x}=\nabla^{2} \bar{\theta}+H(\bar{\varphi}-\bar{\theta}) \\
M \frac{\partial \bar{\varphi}}{\partial t}=\nabla^{2} \bar{\varphi}-\gamma H(\bar{\varphi}-\bar{\theta}) .
\end{gathered}
$$

It can be easily proved that equations (17) - (19) obey the principal of exchange of stabilities and hence the time derivative terms in those equations can be neglected to get

$$
\begin{gathered}
D a\left(\frac{\partial^{2}}{\partial x^{2}}+\frac{\partial^{2}}{\partial y^{2}}\right)^{2} \bar{\psi}-\left(\frac{\partial^{2}}{\partial x^{2}}+\frac{\partial^{2}}{\partial y^{2}}\right) \bar{\psi}+R a \frac{\partial \bar{\theta}}{\partial x}=0, \\
\left(\frac{\partial^{2}}{\partial x^{2}}+\frac{\partial^{2}}{\partial y^{2}}\right) \bar{\theta}+\frac{\partial \bar{\psi}}{\partial x}+H(\bar{\varphi}-\bar{\theta})=0, \\
\left(\frac{\partial^{2}}{\partial x^{2}}+\frac{\partial^{2}}{\partial y^{2}}\right) \bar{\varphi}-\gamma H(\bar{\varphi}-\bar{\theta})=0 .
\end{gathered}
$$

The boundary conditions for the perturbed variables are assumed in the following form

$$
\begin{array}{cc}
\bar{\psi}=0, \quad \frac{\partial^{2} \bar{\psi}}{\partial y^{2}}-D s_{L} \frac{\partial \bar{\psi}}{\partial y}=0, & \frac{\partial \bar{\theta}}{\partial y}-B i_{L} \bar{\theta}=0 \\
\text { and } \quad \frac{\partial \bar{\varphi}}{\partial y}-B i_{L}^{*} \bar{\varphi}=0 & \text { at } \quad y=0, \\
\bar{\psi}=0, \quad \frac{\partial^{2} \bar{\psi}}{\partial y^{2}}+D s_{U} \frac{\partial \bar{\psi}}{\partial y}=0, & \frac{\partial \bar{\theta}}{\partial y}+B i_{U} \bar{\theta}=0 \\
\text { and } \quad \frac{\partial \bar{\varphi}}{\partial y}+B i_{U}^{*} \bar{\varphi}=0 \quad \text { at } \quad y=1 .
\end{array}
$$


Here, $D s_{L}=\alpha_{1} d / \sqrt{K}$ is the slip Darcy number at the lower surface, $D s_{U}=\alpha_{2} d / \sqrt{K}$ is the slip Darcy number at the upper surface, $B i_{L}, B i_{U}$ are the Biot numbers of the fluid phase at lower and upper surfaces respectively, $B i_{L}^{*}, B i_{U}^{*}$ are the Biot numbers of the solid phase at lower and upper surfaces respectively. Equations (20) - (22) admit solutions in the form

$$
\left[\begin{array}{c}
\bar{\psi}(x, y) \\
\bar{\theta}(x, y) \\
\bar{\varphi}(x, y)
\end{array}\right]=\left[\begin{array}{c}
\cos (k x) \psi(y) \\
\sin (k x) \theta(y) \\
\sin (k x) \varphi(y)
\end{array}\right]
$$

where $k$ is the horizontal wave number. By substituting equation (25) into equations (20) - (24) the following set of equations is obtained:

$$
\begin{gathered}
\left\lfloor D a\left(D^{2}-k^{2}\right)-1\right]\left(D^{2}-k^{2}\right) \psi+R a k \theta=0, \\
\left(D^{2}-k^{2}\right) \theta-k \psi+H(\varphi-\theta)=0, \\
\left(D^{2}-k^{2}\right) \varphi-\gamma H(\varphi-\theta)=0, \\
\psi=0, \quad D^{2} \psi-D s_{L} D \psi=0, \quad D \theta-B i_{L} \theta=0 \\
\text { and } \quad D \varphi-B i_{L}^{*} \varphi=0 \quad \text { at } \quad y=0, \\
\psi=0, \quad D^{2} \psi+D s_{U} D \psi=0, \quad D \theta+B i_{U} \theta=0 \\
\text { and } \quad D \varphi+B i_{U}^{*} \varphi=0 \quad \text { at } \quad y=1 .
\end{gathered}
$$

Equations (26) - (30) are solved using advanced successive linearization method by treating $R a$ as an extra variable. The details of the method are discussed in the paper by Narayana et al. [19]. The initial solutions for the advanced successive linearization method were obtained through single-term Galerkin method in the following form:

$$
\left.\begin{array}{l}
\psi(y)=y^{4}-2 a_{1} y^{3}+a_{2} y^{2}+a_{3} y, \\
\theta(y)=y^{2}+a_{4} y-a_{5}, \\
\varphi(y)=y^{2}+a_{6} y-a_{7},
\end{array}\right\}
$$

where, $a_{1}=\frac{12+5 D s_{L}+3 D s_{U}+D s_{L} D s_{U}}{12+4\left(D s_{L}+D s_{U}\right)+D s_{L} D s_{U}}$, 


$$
\begin{aligned}
& a_{2}=\frac{D s_{L}\left(6+D s_{U}\right)}{12+4\left(D s_{L}+D s_{U}\right)+D s_{L} D s_{U}}, \\
& a_{3}=\frac{2\left(6+D s_{U}\right)}{12+4\left(D s_{L}+D s_{U}\right)+D s_{L} D s_{U}}, \\
& a_{4}=\frac{B i_{L}\left(2+B i_{U}\right)}{B i_{U}-B i_{L}-B i_{L} B i_{U}}, \quad a_{5}=\frac{a_{4}}{B i_{L}}, \\
& a_{6}=\frac{B i_{L}^{*}\left(2+B i_{U}^{*}\right)}{B i_{U}^{*}-B i_{L}^{*}-B i_{L}^{*} B i_{U}^{*}}, \quad a_{7}=\frac{a_{6}}{B i_{L}^{*}} .
\end{aligned}
$$

It should be noted here that $\left(D s_{L}, D s_{U}\right) \rightarrow 0$ represents stress-free boundaries and $\left(D s_{L}, D s_{U}\right) \rightarrow \infty$ represents rigid boundaries. Similarly, the case $\left(B i_{L}, B i_{U}\right) \rightarrow 0$ represents adiabatic type temperature boundary condition while $\left(B i_{L}, B i_{U}\right) \rightarrow \infty$ represents isothermal type boundary condition. Other combinations of boundary conditions are obtained for some particular choice of values of these parameters.

\section{RESULTS AND DISCUSSION}

A semi-analytical study is made of the linear stability analysis for the RayleighBénard-Brinkman convective instability problem that assumes local thermal nonequilibrium between the fluid and solid phases and that considers general boundary conditions on velocity and temperatures. The governing equations result in a twopoint eigen boundary value problem with third type boundary conditions. It is the boundary conditions that pose obstacles in obtaining an analytical expression for the eigen value $R a_{c}$ (critical Rayleigh number). The most general formulation of the problem facilitates the handling of 2 limiting case problems which at the present time are being tackled as 2-independent problems. The integrated approach to the convective instability problem gives a new direction to the investigation of such problems. The main difficulty in solving such EBVP's with generalized boundary conditions concerns convergence of the schemes, iterative or otherwise.

In this paper we propose the advanced successive linearization method (ASLM). The method when used in the original form does pose problems in the case of general boundary conditions. The usual problems encountered are the following:

1. Sensitivity of the convergence of the solution to the choice of initial unknown guess values and eigen values.

2. Extreme values of the parameters many a time slow down convergence and warrant use of acceleration procedures. 
3. Greater accuracy if required for the eigen values would mean greater the intensity of the above two problems.

4. Choice of basis functions also makes contributions to all the three computational problems noted above.

In the present problem we have circumvented all the above hurdles by adopting the following scientific procedure:

The elegance in the ASLM method can be appreciated only when it is implemented in an automated way in the chosen computer program. Since MATLAB implements algorithms mostly through matrices, the ASLM based procedure that leans heavily on matrix based concepts is ideally suited for Chebyshev-based spectral ASLM method. SciLab is another such package (open source) that provides natural support for implementation of ASLM procedure.

These results are in line with those reported by Siddheshwar [18]. Also, the critical Raleigh number corresponding to the Robin type temperature boundary conditions lies in between those of isothermal and adiabatic type of temperature boundary conditions, i.e.,

$$
\left(R a_{c}\right)_{\text {Adiabatic }} \leq\left(R a_{c}\right)_{\text {Robin }} \leq\left(R a_{c}\right)_{\text {Isothermal }} \text {. }
$$

Table 1: Comparision of Rc and Kc obtained from the present study with those obtained by A. Postelnicu and D. A.S. Rees (2003) for the limiting case $\left(10^{\wedge} 3,1\right)$

\begin{tabular}{|c|c|c|c|c|c|}
\hline $\begin{array}{c}\text { Boundary } \\
\text { Combination }\end{array}$ & & \multicolumn{2}{|c|}{ Postelnicu \& Rees } & \multicolumn{2}{|c|}{ Present Result } \\
\cline { 3 - 6 } & & \multicolumn{2}{|c|}{ Rc } & Kc & Rc \\
\hline FIFI & $\begin{array}{c}\text { Log } \\
\text { H }\end{array}$ & Kc & Rc & & \\
\hline & 1.5 & 3.36726 & 64.75793 & 3.36726 & 64.75793 \\
\hline & 2 & 3.23474 & 73.81888 & 3.23474 & 73.82699 \\
\hline & 2.5 & 3.15609 & 78.14846 & 3.15609 & 78.14846 \\
\hline & 3 & 3.12642 & 79.73422 & 3.12642 & 79.74002 \\
\hline & 3.5 & 3.11654 & 80.26042 & 3.11654 & 80.26273 \\
\hline & 4 & 3.11337 & 80.4294 & 3.11337 & 80.43092 \\
\hline & 4.5 & 3.11236 & 80.4831 & 3.11236 & 80.4844 \\
\hline & 5 & 3.11204 & 80.50011 & 3.11204 & 80.50134 \\
\hline
\end{tabular}


Table 2: Comparison of $\mathrm{Rc}$ and kc obtained from the present $\left(\mathrm{Da}=10^{\wedge}(-2), \gamma=1\right)$

\begin{tabular}{|c|c|c|c|c|c|c|c|}
\hline & RIRI & & $\begin{array}{c}\text { RARI } \\
\text { (same as } \\
\text { RIRA })\end{array}$ & & & $\begin{array}{c}\text { RAFI } \\
\text { (same as } \\
\text { FIRA) }\end{array}$ & \\
\hline $\begin{array}{c}\text { Log } \\
\text { H }\end{array}$ & Kc & Rc & Kc & Rc & Rc & Kc & Rc \\
\hline-2 & $\begin{array}{c}3.2317 \\
4\end{array}$ & 60.42749 & 2.51257 & $\begin{array}{c}45.3053 \\
9\end{array}$ & 53.15413 & 2.40667 & $\begin{array}{c}39.9040 \\
6\end{array}$ \\
\hline-1 & $\begin{array}{c}3.2381 \\
1\end{array}$ & 60.69288 & 2.53233 & $\begin{array}{c}45.7259 \\
4\end{array}$ & 53.39958 & 2.42552 & $\begin{array}{c}40.2920 \\
1\end{array}$ \\
\hline 0 & $\begin{array}{c}3.2938 \\
1\end{array}$ & 63.20008 & 2.67594 & $\begin{array}{c}49.3449 \\
6\end{array}$ & 55.71402 & 2.56093 & $\begin{array}{c}43.6140 \\
6\end{array}$ \\
\hline 1 & $\begin{array}{c}3.4945 \\
4\end{array}$ & 79.74521 & 2.91002 & $\begin{array}{c}66.5124 \\
8\end{array}$ & 70.77283 & 2.76253 & $\begin{array}{c}59.0170 \\
6\end{array}$ \\
\hline 2 & $\begin{array}{c}3.3519 \\
7\end{array}$ & 110.3805 & 2.61038 & $\begin{array}{c}\mathbf{8 5 . 9 2 3 8} \\
1\end{array}$ & $\mathbf{9 7 . 4 9 4 1 4}$ & 2.49107 & $\begin{array}{c}75.8010 \\
6\end{array}$ \\
\hline 3 & $\begin{array}{c}3.2456 \\
2\end{array}$ & 119.5794 & 2.52118 & $\begin{array}{c}90.0112 \\
2\end{array}$ & 105.2378 & 2.4139 & $\begin{array}{c}79.2907 \\
8\end{array}$ \\
\hline
\end{tabular}

In the present problem, the non-boundary parameters that affect the critical Rayleigh number are the Darcy number $D a$, the inter-phase heat transfer parameter $H$ and the porosity-scaled conductivity ratio $\gamma$. We now discuss effects of these parameters on the onset of convection with upper and lower stress-free-isothermal (FIFI) boundaries and upper and lower rigid-isothermal (RIRI) boundaries. Table 1 gives the critical Rayleigh number and the critical wave number for different values of $D a, \gamma$ and $\log H$. It is clear that increasing values of $\log H$ results in increasing the critical Rayleigh number indicating that the inter-phase heat transfer parameter has a stabilizing effect on the onset of convection in the case of stress-free-isothermal (FIFI) and rigid-isothermal (RIRI) boundaries. It is observed that the critical Rayleigh number is a decreasing function of the parameter $\gamma$ which mean that convection sets in much earlier than expected for higher values $\gamma$ vowing to its destabilizing effect in both FIFI and RIRI cases. Further, the Darcy number also increases the critical Rayleigh number in FIFI and RIRI case. Hence it has a stabilizing effect on the system considered. Results of the other mixed combinations of boundary conditions like rigid-stress-free, isothermal-adiabatic are obtained as special cases of the general boundary conditions considered in the paper. We omit the discussion of the same for reasons of space. 


\section{CONCLUSIONS}

In this paper we presented a semi-analytical study of the linear stability analysis for the Rayleigh-Bénard-Brinkman convective instability problem that assumes local thermal non-equilibrium between the fluid and solid phases and that considers general boundary conditions on velocity and temperatures. Table 1, Comparision of Rc and Kc obtained from the present study with those obtained by A. Postelnicu and D. A.S. Rees (2003) and Table 2: Comparison of $\mathrm{Rc}$ and $\mathrm{kc}$ obtained from the present $\left(\mathrm{Da}=10^{\wedge}(-2), \quad \gamma=1\right)$. The eigen boundary value problem was solved using the advanced successive linearization method with the help of single-term Galerkin method for initial solutions. The analysis predicts the critical Rayleigh number corresponding to the third kind temperature boundaries to be between those of adiabatic and isothermal cases. Further, the Darcy number and the inter-phase heat transfer parameter have stabilizing effect on the system while the opposite is true in the case of porosity-scaled conductivity ratio.

\section{REFERENCES}

[1] C.W. Horton and F.T. Rogers, "Convection currents in a porous medium", Journal of Applied Physics 16, 367-370 (1945).

[2] E.R. Lapwood, "Convection of a fluid in a porous medium", Proceeding of the Cambridge Philosophical Society 44, 508-521 (1948).

[3] D.B. Ingham and I. Pop, Transport phenomena in porous media (Pergamon, Oxford, 1998).

[4] D.A. Nield and A. Bejan, Convection in porous media (Springer-Verlag, New York, 1999).

[5] K. Vafai, Handbook of porous media (Marcel Dekker, New York, 2000).

[6] N. Banu and D.A.S. Rees, "Onset of Darcy-Bénard convection using a thermal nonequilibrium model", International Journal of Heat and Mass Transfer 45, 2221-2228 (2002).

[7] A. Postelnicu and D.A.S. Rees, "The onset of a Darcy-Brinkman convection using a thermal nonequilibrium model-Part I: stress-free boundaries", International Journal of Energy Research 27, 961-973 (2003).

[8] M.S. Malashetty, I.S. Shivakumara and S. Kulkarni, "The onset of LapwoodBrinkman convection using a thermal non-equilibrium model", International Journal of Heat and Mass Transfer 48, 1155-1163 (2005).

[9] A. Nouri-Borujerdi, A.R. Noghrehabadi and D.A.S. Rees, "The effect of local thermal non-equilibrium on conduction in porous channels with a uniform heat 
source", Transport in Porous Media 69, 281-288 (2007).

[10] A. Postelnicu, "The onset of a Darcy-Brinkman convection using a thermal nonequilibrium model. Part II", International Journal of Thermal Sciences 47, 1587-1594 (2008).

[11] M.S. Malashetty, I.S. Shivakumara and S. Kulkarni, "The onset of convection in a couple stress fluid saturated porous layer using a thermal non-equilibrium model", Physics Letters A 373, 781-790 (2009).

[12] I.S. Shivakumara, M.S. Malashetty and K.B. Chavaraddi, Onset of convection in a viscoelastic fluid-saturated sparsely packed porous layer using a thermal nonequilibrium model, Candian Journal of Physics 84, 973-990 (2006).

[13] M.S. Malashetty, M. Swamy, R. Heera, Double diffusive convection in a porous layer using a thermal non-equilibrium model, International Journal of Thermal Sciences 47, 1131 - 1147 (2008).

[14] M.S. Malashetty and R. Heera, "Linear and non-linear double diffusive convection in a rotating porous layer using a thermal non-equilibrium model", International Journal of Non-Linear Mechanics 43, 600-621 (2008).

[15] M.S. Malashetty, I. Pop and R. Heera, "Linear and nonlinear double diffusive convection in a rotating sparsely packed porous layer using a thermal nonequilibrium model", Continuum Mechanics and Thermodynics 21, 317-339 (2009).

[16] M.S. Malashetty, I.S. Shivakumara and S. Kulkarni, "The onset of convection in an anisotropic porous layer using a thermal non-equilibrium model", Transport in Porous Media 60, 199-215 (2005).

[17] I.S. Shivakumara, J. Lee, A.L. Mamatha and M. Ravisha, "Boundary and thermal non-equilibrium effects on convective instability in an anisotropic porous layer", Journal of Mechanical Science and Technology 25, 911-921 (2011).

[18] P.G. Siddheshwar, "Convective instability of ferromagnetic fluids bounded by fluid-permeable, magnetic boundaries", Journal of Magnetism and Magnetic Materials 149, 148-150 (1995).

[19] M. Narayana, S.S. Motsa and P. Sibanda, "On a piecewise linearization solution of an eigen BVP and chaotic system due to magneto-convection in a two dimensional rectangular box", to be communicated (2014). 
